\title{
Convective and absolute Eckhaus instability leading to modulated waves in a finite box
}

\author{
Nicolas Garnier,f Arnaud Chiffaudel,f and François Daviaud \\ Service de Physique de l'État Condensé, DSM, CEA Saclay, CNRS SNC 2005, 91191 Gif-sur-Yvette, France
}

(Published in Phys. Rev. Lett. 88 (13) 134501 April 2002)

\begin{abstract}
We report experimental study of the secondary modulational instability of a one-dimensional nonlinear traveling wave in a long bounded channel. Two qualitatively different instability regimes involving fronts of spatio-temporal defects are linked to the convective and absolute nature of the instability. Both transitions appear to be subcritical. The spatio-temporal defects control the global mode structure.
\end{abstract}

PACS numbers: 47.20.Lz, 47.35.+i, 47.54.+r, 05.45.-a

The Eckhaus instability [1] is one of the major secondary instability of nonlinear patterns. While the Eckhaus dynamics for steady patterns (e.g., RayleighBénard) is fast, dealing with local wavelength nucleation or annihilation [2], it presents slow evolution of traveling modulated waves in the traveling-wave-pattern case. Such modulated patterns are believed to be essential for the description of the transition to phase and defect chaos in complex Ginzburg-Landau models [3]. Experiments on nonlinear traveling waves are frequently carried out in annular cells [ [ , 5, 6, 7, 8] for the simplicity of the underlying wave pattern, and generally consider the Eckhaus instability close to the wave threshold. The main specificity of our wave-system is to become Eckhaus unstable for increasing value of the control parameter, i.e., as a first step on the route to spatio-temporal chaos [8]. In this Letter, we focus on new results in a long rectangular cell: an homogeneous traveling-wave undergoing the Eckhaus instability generates modulated waves. Our results reveal the rich effect of a finite group velocity within a closed cell: we describe quantitatively the convective and absolute modulated wave-patterns and the associated transitions.

Setup. Our physical results concern the secondary bifurcation of a wave pattern. We treat this system as a nonlinear wave model: this approach does not require any connection with the underlying physics of the convective flow. The experimental setup, its basic flow and the nature of the primary bifurcation producing the underlying wave-pattern have been described in detail [9]. It consists of a thermocapillary convective flow in a long narrow channel where an external parameter - the horizontal temperature difference $\Delta T$ - drives an instability toward propagating hydrothermal waves [9, 10, 11]. The length of the channel is $L=180 \mathrm{~mm}$. It is occasionally compared to an equivalent annular channel [8] of perimeter $P=503 \mathrm{~mm}$, i.e., a periodic boundary condition system. The aspect ratios ensure one-dimensional patterns.

We have shown [9] this primary bifurcation to be well described by the convective/absolute transition: a global mode is the first structure observed when the control parameter $\Delta T$ is increased above the absolute threshold $\Delta T_{\mathrm{a}}=3.66 \mathrm{~K}$. In the periodic channel, waves ap- pear at the convective threshold $\Delta T_{\mathrm{c}}=3.1 \mathrm{~K}$ which can be used to build a dimensionless control parameter $\epsilon=\Delta T / \Delta T_{\mathrm{c}}-1$. Above complex competition regimes between right- and left-traveling waves (e.g. blinking states), we have shown a single wave train to become almost uniform in the cell for $\Delta T \gtrsim 4.5 \mathrm{~K}$ (Fig. 1). This state constitutes the basic state for the present study: we will now focus on the secondary instability of this single wave train.

When $\Delta T$ is increased far enough from the primary onset, a modulational instability occurs. As the group velocity is finite, the modulational perturbations are advected. The present paper focuses on the distinction between the convective and the absolute modulational instability regimes and the relevance of a new object: a front of dislocations or modulations. In periodic conditions, this modulational instability occurs at the lowest possible wavenumber $K_{\bmod }=2 \pi / P$ [8]: it is strictly an Eckhaus [1] instability. In the linear channel, for simplicity, we will also refer to Eckhaus instability, although the wavenumber of the modulational instability modes are somehow larger: typically $K_{\text {mod }} \sim 4 \cdot(2 \pi / L)$.

Absolute instability. Figs 2 and 3 present the three states which support our discussion. For $\Delta T>\Delta T_{\mathrm{m}, \mathrm{a}}=$ $(5.56 \pm 0.03) \mathrm{K}$, the observed pattern (Fig. 2b) can be described as a wave composed of two wave trains of mean wavenumbers $k_{\mathrm{u}}$ and $k_{\mathrm{d}}$. The wavenumber, frequency and amplitude of both wave trains are modulated in space and time. The wavenumber $K_{\bmod }$ of the modulation is of order of $\left|k_{\mathrm{u}}-k_{\mathrm{d}}\right|$. Waves are emitted from one end of the cell with wavenumber $k_{\mathrm{u}} \sim 21 \cdot(2 \pi / L)$ and

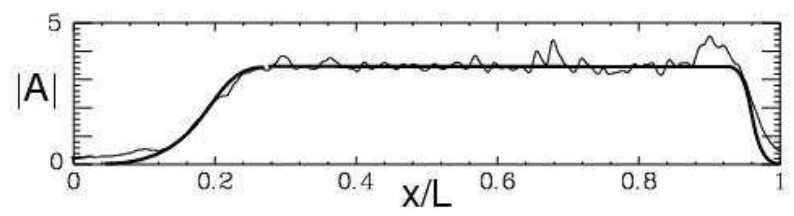

FIG. 1: The measured (thin line) and schematic (thick line) amplitude profile of the single-wave system for $\Delta T=4.75 \mathrm{~K}$. The pattern is presented as the envelope amplitude $A(x)$ of a right-traveling wave, although both directions are equivalent. The amplitude $B(x)$ of the minor (left) wave is negligible. For details see Fig. 2 in Ref. [9]. 


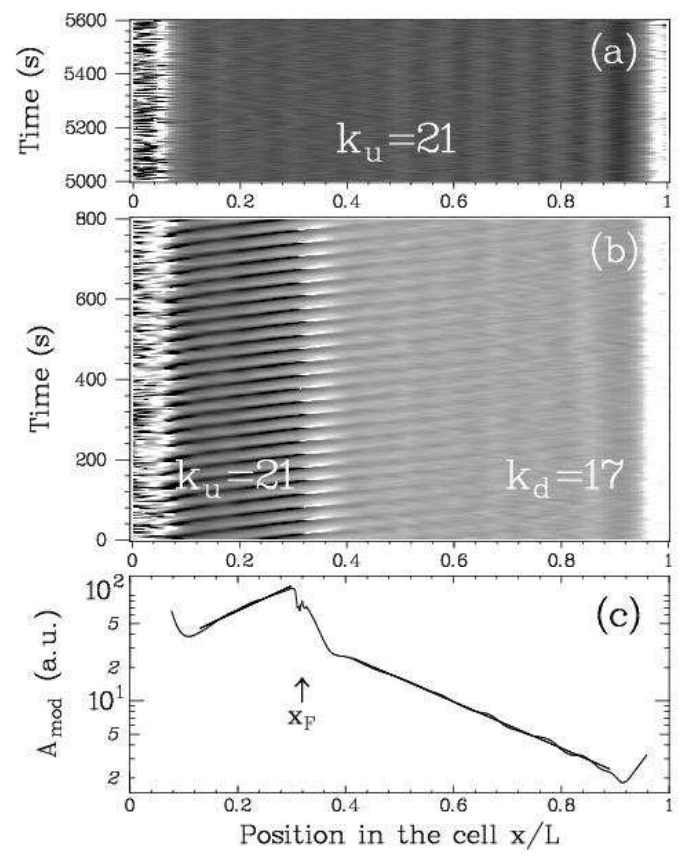

FIG. 2: Spatio-temporal diagrams of the local and instantaneous wavenumber $k(x, t)$ of the wave: temporally stabilized regimes for (a) $\Delta T=5.54 \mathrm{~K}$ and (b) $5.65 \mathrm{~K}$. The waves propagate from left to right. The mean wavenumber can be visually estimated by the mean gray level and is labeled (units $2 \pi / L$ ) in the upstream $\left(k_{\mathrm{u}}\right)$ and downstream $\left(k_{\mathrm{d}}\right)$ regions. A uniform wavenumber (a) represents a non-bifurcated state, and illustrates both stable and convective regimes below $\Delta T_{\mathrm{m}, \mathrm{a}}$. The modulated state (b) is the global mode of the Eckhaus instability. Each black to white transition of the wavenumber value at $x_{F} / L=0.32$ is due to a phase jump in the core of a defect. The defect front is stable with time. (c) By Hilbert demodulation of phase-gradient image (b) we get the spatial profile of the amplitude $A_{\bmod }$ of the modulation.

propagate along the cell at the phase velocity. The phase modulation of this wave train, traveling at the group velocity, is spatially growing. On Fig. 2ac, we clearly see the exponential growth of the local-wavenumber modulation amplitude $A_{\text {mod }}$ along $x$. At a fixed, finite distance $x_{F}$ from the source-boundary, the wavenumber modulation is so large that it allows the wavenumber to change from $k_{\mathrm{u}}$ to $k_{\mathrm{d}}$ by time-periodic phase slips. For $x>x_{F}$, the mean wavenumber is $k_{\mathrm{d}} \sim 17 \cdot(2 \pi / L)$. In this second region, the modulation is damped (Fig. 2b,c): we conclude that $k_{\mathrm{u}}\left(\right.$ resp. $\left.k_{\mathrm{d}}\right)$ waves are unstable (resp. stable) with respect to modulations.

We call dislocation front the set of spatio-temporal loci where spatio-temporal dislocations occur. For $\Delta T>$ $\Delta T_{\mathrm{m}, \mathrm{a}}$, the position $x_{F}$ of this object is stationary; Fig. 1 shows the relation between the control parameter and the front position which remains located in the first half of the cell whatever the value of $\Delta T$. Steady dislocation fronts have been observed for traveling waves in a TaylorDean experiment [12]. In general, hysteresis has not been investigated [13]. From the modulation amplitude profiles $A_{\text {mod }}(x)$ (Fig. 2 $c$ c), we also extract the spatial growth

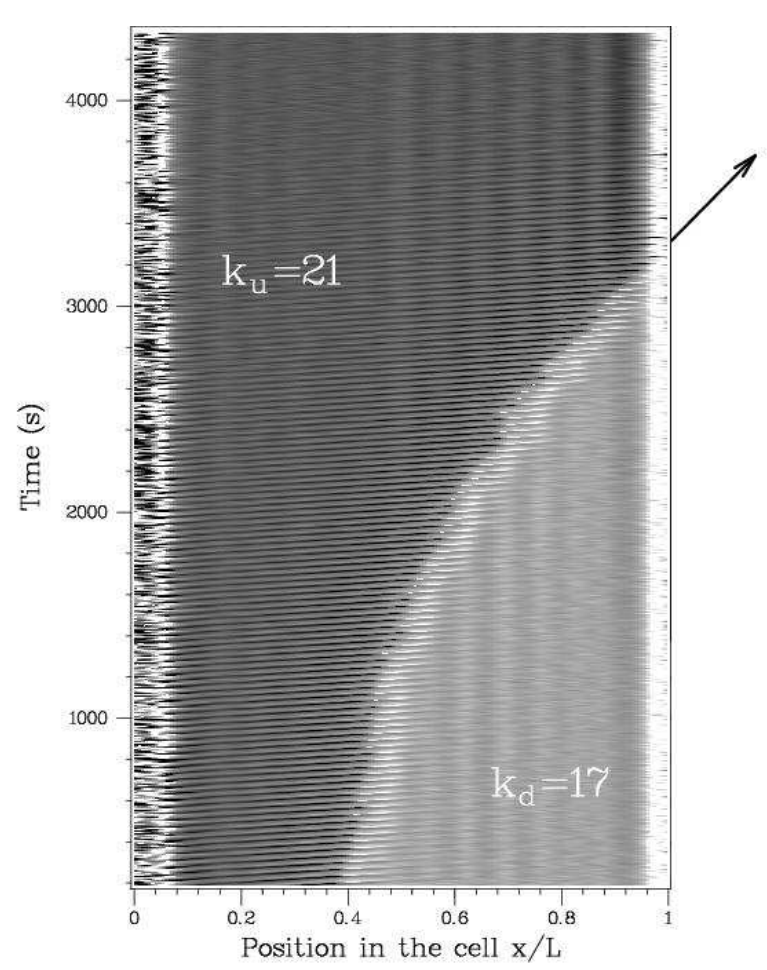

FIG. 3: Transient leading to the state of Fig. 2a. The state has been prepared at $t=0$ : a dislocation front is slowly advected out of the cell. The modulations grow along $x$ but vanish along $t$ : this is the signature of a convective instability regime. The arrow indicates the asymptotic front velocity.

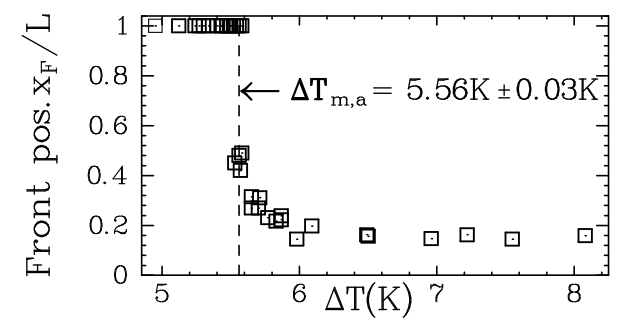

FIG. 4: Spatial position $x_{F}$ of the dislocation front for absolutely unstable states vs $\Delta T$. Stable and convectively unstable states without permanent dislocation front are represented by a symbol at $x_{F}=L$.

rate of the modulations: it is finite and positive (squares on Fig. 可a).

We claim that those stationary states to be the global modes for the modulational (Eckhaus) instability. The structure of these global modes is very peculiar: nothing seems to saturate the modulations except the breakup of the underlying wave-pattern, i.e., the abrupt change of the mean-wavenumber downstream the dislocations. Similar patterns have been numerically observed in semiinfinite 114 and closed cells [15]. Like Couairon and Chomaz [14] we observe the nonlinear global threshold and the absolute instability threshold to be identical.

Convective instability. For $\Delta T<\Delta T_{\mathrm{m}, \mathrm{a}}$, dislocation fronts are not observed on asymptotic states. The asymptotic regime (Fig. 2a) is an homogeneous wave of uni- 
form wavenumber $k_{\mathrm{u}} \sim 21 \cdot(2 \pi / L)$. However, transients obtained after control parameter changes show traveling dislocation fronts slowly advected out of the channel (Fig. 3): those states are convectively unstable states with respect to the modulational (Eckhaus) instability. They are observed in the small gap between $\Delta T_{\mathrm{m}, \mathrm{c}}=5.45 \mathrm{~K}$ and $\Delta T_{\mathrm{m}, \mathrm{a}}=5.56 \mathrm{~K}$.

For $\Delta T<\Delta T_{\mathrm{m}, \mathrm{c}}$, asymptotic states are uniform and dislocation fronts do not exist. Close to $\Delta T_{\mathrm{m}, \mathrm{c}}$ very long transients are often observed. These transient patterns (not shown) are also slightly modulated; the modulations do not reach the critical amplitude producing dislocations; the modulation amplitude profiles generally decrease (negative spatial growth rate) along the downstream direction and slowly travel toward the upstream direction. So, the uniform wave looks stable. The transients may last much longer than the experimental running time, and those results have to be considered with care.

Using a second Hilbert transform of phase-gradient data (as Fig. 2 $\mathrm{c}$ ), we measured spatial and temporal growth rates of the modulation. We present these data for the unstable upstream wave train. The temporal growth rate for modulations in the laboratory frame is negative below $\Delta T_{\mathrm{m}, \mathrm{a}}$ and positive above. It is also close to zero around the convective transition where very long transients are observed. The spatial growth rate of the upstream $k_{\mathrm{u}}$ wave train for all three regimes is presented on Fig. Fa. It is positive for both unstable regimes but the slope is seemingly different in the convective and absolute cases. It is negative below $\Delta T_{\mathrm{m}, \mathrm{c}}$.

Perturbed states. In order to test the above description, we perturbed the uniform states either by plunging a thin needle in the convective layer or by dropping a cold or hot droplet of fluid. The frequency content of those perturbations differs from the above reported transients: the modulation wave trains contain only a few wavelengths and appear to be advected downstream at roughly the group velocity. All observed perturbations show positive spatial growth rate and negative temporal growth rate in the laboratory frame. The spatial growth rates are presented on Fig. $5 \mathrm{~b}$. In the convective regime, the growth rate appears to be selected at the same value as in spontaneous transients. In the stable regime, however, the data are very dispersed but remain positive.

Discussion. Let us start our discussion by two important remarks:

(i) The modulation amplitude $A_{\bmod }$ never saturates: All observed $A_{\text {mod }}$ profiles appear locally exponential along $x$. No nonlinear saturation effect is thus observed. The occurrence of dislocations (for $A_{\bmod } \sim\left|k_{\mathrm{u}}-k_{\mathrm{d}}\right|$ ) is the only limit to exponential growth. This is a strong argument for the Eckhaus instability to behave subcritically in this closed cell. Remember it is supercritical in the annular cell [8]. This difference is due to the mean wavenumber of the carrier-wave pattern. It will be dis-

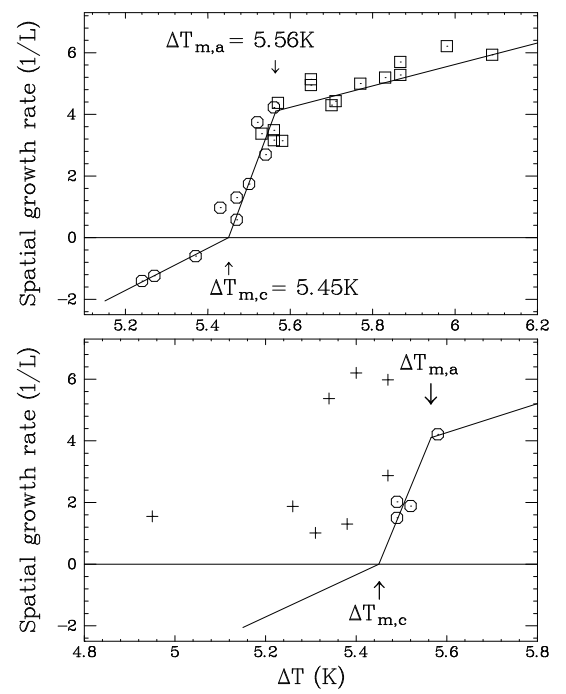

FIG. 5: (a) Evolution of the spatial growth rate of the modulation with the control parameter for transient (O) or steady (squares) spontaneously modulated wave patterns. Linear fits of the three regimes - stable, convective and absolute - are presented. They intersect at $\Delta T_{\mathrm{m}, \mathrm{c}}$ and $\Delta T_{\mathrm{m}, \mathrm{a}}$. These data concern the modulations of the upstream region of the cell whose mean wavenumber is $k_{\mathrm{u}} \sim 21 \cdot(2 \pi / L)$. Corresponding data for the downstream region are negative while $\Delta T \lesssim 8 \mathrm{~K}$. (b) Idem for perturbation initiated wave-packets in the stable $(+)$ and convectively unstable (O) regimes. The solid lines reproduce the fits of (a) to allow quantitative comparisons: the same growth rate is selected in both cases for the convective regime.

cussed elsewhere.

(ii) Reflections: The modulation wave system is a perfect single wave system: the reflections of the modulations at the boundaries are irrelevant since there is no possibility for reflected information to travel back to the source.

The observation facts described above are coherent with the interpretation in terms of convective and absolute instability. The striking point is the positive spatial growth rates for perturbations in the seemingly stable regime below $\Delta T_{\mathrm{m}, \mathrm{c}}$. As for spontaneously modulated patterns, we would expect those modulation wavepackets to decrease in space exactly as the stable $k_{\mathrm{d}}$ wave trains do in the absolute regime (Fig. 2 2 c).

Suppose that the convective instability is subcritical as suggested in remark (i). Then, above $\Delta T_{\mathrm{m}, \mathrm{c}}$, the transient evolves on an unstable branch (Fig. 3) close to the absolute branch (Fig. 2b). However, below $\Delta T_{\mathrm{m}, \mathrm{c}}$, a second unstable branch co-exists, which can be reached only by perturbing the flow: this description can be supported by the schematic Fig. 6 inspired by zero group velocity instabilities. These branches present very different patterns: The upper branch exhibits extended modulations over the whole cell, with slow evolution and, for high enough amplitudes - the generally observed case above $\Delta T_{\mathrm{m}, \mathrm{c}}-$, dislocation fronts. The lower branch exhibits 


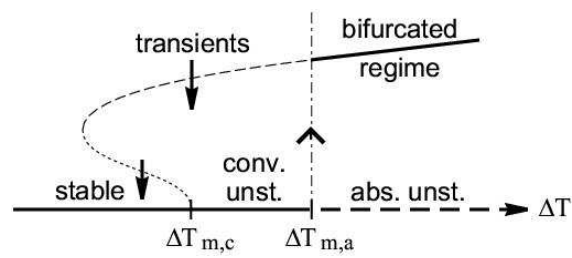

FIG. 6: Schematic representation of the observed regimes, based on the usual representation of a subcritical bifurcation with zero group velocity. The ordinate is only qualitative. Solid heavy lines represent the steady states, bifurcated or not, above or below $\Delta T_{\mathrm{m}, \mathrm{a}}=5.56 \mathrm{~K}$. The thin dashed lines may account for two different transient modes (see text).

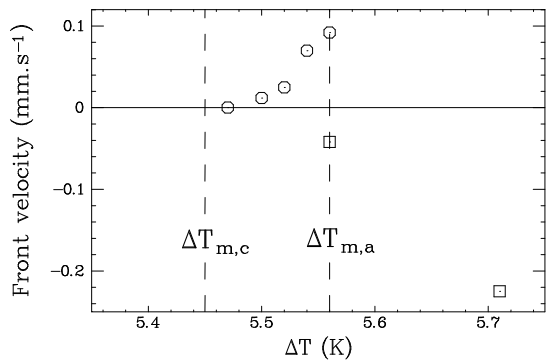

FIG. 7: Front velocity around the convective/absolute transition. The circles $(0)$ show the velocity of dislocation fronts in transient convective regimes below $\Delta T_{\mathrm{m}, \mathrm{a}}$. Above $\Delta T_{\mathrm{m}, \mathrm{a}}$, the (negative) velocities of transient modulation fronts invading the cell from downstream are shown by squares. For comparison, the group velocity at wave onset is $0.90 \mathrm{~mm} . \mathrm{s}^{-1}$.

fast-traveling narrow modulation wave trains and cannot be reached spontaneously by varying $\Delta T$. This hypothesis can explain the very different aspect of spontaneous and induced transients in the stable regime below $\Delta T_{\mathrm{m}, \mathrm{c}}$. It is also known that the shape of induced nonlinear patterns below subcritical instabilities depends of the forcing amplitude [16], so the dispersion in Fig. 5b may be due to both the effect of amplitude and the presence of the two branches.

Another observation of the convective branch is intriguing. We record the asymptotic velocity of the dislocation fronts between $\Delta T_{\mathrm{m}, \mathrm{c}}$ and $\Delta T_{\mathrm{m}, \mathrm{a}}$, i.e., the tangent to the space-time trajectory when the front leaves the cell (Fig. 7). The observation is surprising: the closer we are to the absolute instability onset, the faster the front moves! Then its velocity jumps below zero above $\Delta T_{\mathrm{m}, \mathrm{a}}$. A contrario, around $\Delta T_{\mathrm{m}, \mathrm{c}}$, the front velocity is zero, leading to infinitely long transients, i.e., temporal marginality. This quantifies the experimental complexity of carrying out the experiment around this point. What is the meaning of the velocity jump at $\Delta T_{\mathrm{m}, \mathrm{a}}$ ? Is the convective/absolute transition also subcritical? It is probably: while our protocol [13] did not allow to explore all branches by varying $\Delta T$ up and down from one state to another, a test has been made to transit directly from an absolute state to a stable state just below $\Delta T_{\mathrm{m}, \mathrm{c}}$ : the absolute modulation profile remains fixed in the cell. This can be due either to hysteresis, or to the vanishing front velocity... which makes the system marginal in this region. This point would need to be addressed with an improved experimental device.

Finally, in some regimes to be presented elsewhere [17], the front position $x_{F}(t)$ exhibits chaotic behaviors (period doubling or quasiperiodicity): it can thus be viewed as the order parameter for the modulational instability up to the transition to spatio-temporal chaos.

To conclude, we claim to have observed both convective and absolute transitions for a modulational or Eckhaus instability in a long bounded channel. The subcritical convective transition is characterized by zero spatial growth rate and zero advection velocity for the modulated wave pattern, which can be viewed as spatial and temporal marginality. The absolute transition is characterized by the dynamics of dislocation fronts. The front velocity data suggest the transition to be subcritical as well. This question deserves a theoretical support which remains, to our knowledge, unexplored.

We thank A. Casner and C. Gasquet who participated to the data collection and J.M. Chomaz, J.M. Flesselles and P. Manneville for fruitful discussions.

* Present address: Center for Nonlinear Science, School of Physics, Georgia Institute of Technology, Atlanta, Georgia $30332-0430$.

† Electronic address: arnaud.chiffaudel@cea.fr

[1] W. Eckhaus, Studies in Nonlinear Stability Theory, Springer, New York (1965).

[2] L. Kramer \& W. Zimmermann, Physica D 16, 221 (1985).

[3] L. Brusch, M.G. Zimmermann, M. van Hecke, M. Bär \& A. Torcini, Phys. Rev. Lett., 85, 86 (2000). L. Brusch, A. Torcini, M. van Hecke, M.G. Zimmermann \& M. Bär, Physica D 160, 127 (2001).

[4] B. Janiaud, A. Pumir, D. Bensimon, V. Croquette, H. Richter \& L. Kramer, Physica D 55, 269 (1992).

[5] P. Kolodner, Phys. Rev. A 46, 6431 (1992).

[6] T. Leweke \& M. Provansal, J. Fluid Mech. 288, 265 (1995).

[7] Y. Liu \& R.E. Ecke, Phys. Rev. E 59, 4091 (1999).

[8] N. Mukolobwiez, A. Chiffaudel \& F. Daviaud, Phys. Rev. Lett., 80, 4661 (1998).

[9] N. Garnier \& A. Chiffaudel, Phys. Rev. Lett., 86, 75 (2001).

[10] F. Daviaud \& J.M. Vince, Phys. Rev. E 48, 4432 (1993).

[11] J. Burguete, N. Mukolobwiez, F. Daviaud, N. Garnier \& A. Chiffaudel, Phys. Fluids, 132773 (2001).

[12] P. Bot \& I. Mutabazi, Eur. Phys. J. B 13, 141 (2000).

[13] In order to get long data series close to onsets, we often used the following protocol: the temperature gradient is first established in the vessel, the cell is refilled and the fluid is agitated to break the thermal gradients. Within a few seconds the hydrothermal waves reappear without history.

[14] A. Couairon \& J-M. Chomaz, Physica D, 132, 428 (1999).

[15] S.M. Tobias, M.R.E. Proctor \& E. Knobloch, Physica D 
113, 43 (1998).

[16] S. Bottin \& H. Chaté, Eur. Phys. J. B 6, 143 (1998).

[17] N. Garnier, A. Chiffaudel and F. Daviaud, to be pub- lished in Physica D. 